\title{
COVID-19 em pacientes com câncer: gerenciamento de uma pandemia dentro de uma pandemia.
}

\author{
Karina Figueira (1)
}

\section{EDITORIAL}

\section{Resumo}

A pandemia da doença coronavírus 2019 (COVID-19) interrompeu os cuidados de saúde em todo o mundo. Pacientes com câncer parecem ser particularmente suscetíveis às morbidades e mortalidade dessa nova doença. Nenhuma terapia específica para COVID-19 atualmente parece oferecer um benefício de sobrevida para essa população de pacientes. Além disso, os efeitos globais no tratamento de rotina do câncer provavelmente só serão sentidos nas próximas décadas.

Palavras Chave: Covid 19, Sars-Cov-2, Câncer, Oncologia, Pandemia, Coronavírus. 


\section{COVID-19 in cancer patients: managing a pandemic within a pandemic.}

The 2019 coronavirus disease pandemic (COVID-19) has disrupted health care worldwide. Cancer patients appear to be particularly susceptible to the morbidity and mortality of this new disease. No specific therapy for COVID-19 currently appears to offer a survival benefit for this patient population. In addition, the global effects on routine cancer treatment are likely to be felt only in the coming decades.

Keywords: Covid 19, Sars-Cov-2, Cancer, Oncology, Pandemic, Coronavirus.

Instituição afiliada: 1- Grupo de Odontologia Especializada, Macapá, Amapá, Brasil.

Dados da publicação: Artigo recebido em 10 de Novembro, revisado em 15 de Novembro, aceito para publicação em 19 de Novembro e publicado em 29 de Novembro.

DOI: https://doi.org/10.36557/2674-8169.2020v2n12p01-06

@ Karina Figueira odontofigueira@yahoo.com.br

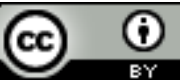

This work is licensed under a Creative Commons Attribution 4.0 International

License. 


\section{COVID 19 e Pacientes oncológicos}

Doença por coronavírus 2019 (COVID-19), uma infecção do trato respiratório causada pela síndrome respiratória aguda grave coronavírus denominada SARS-CoV-2, surgiu inicialmente na China no final de 2019. A rápida disseminação global desse novo vírus levou a OMS a declarar um pandemia com> 30.000.000 de casos confirmados, 946.000 mortes e> 21.000.000 de recuperações relatadas em 18 de setembro de 2020, de acordo com o Johns Hopkins Coronavirus Resource Center .

Relatórios iniciais da Ásia sugeriram que pacientes idosos com múltiplas comorbidades, especificamente diabetes, hipertensão e obesidade, tinham um risco aumentado de desenvolver COVID-19 grave após infecção por SARS-CoV-2 ${ }^{1,2}$. À medida que os dados sobre esses riscos evoluíram, as evidências mostram cada vez mais que os pacientes com câncer são, de fato, um grupo particularmente vulnerável. No entanto, os efeitos de vários fatores de confusão, incluindo uma população de pacientes mais velha do que a média, que muitas vezes têm comorbidades subjacentes, incluindo um sistema imunológico suprimido e / ou um estado hipercoagulável, têm sido difíceis de separar dos efeitos de ter câncer. Igualmente confusos para os médicos são os sintomas comuns de apresentação da SARS-CoV-2, incluindo dispneia, tosse, febre, fadiga, disgeusia e, menos comumente, diarreia e / ou uma síndrome hiperinflamatória, que são todos sintomas de apresentação comuns de câncer e toxicidades do câncer terapia. Além disso ${ }^{3}$. Os primeiros relatórios que descrevem resultados em pacientes com COVID-19 incluíram menos de um punhado de pacientes com câncer na Ásia ${ }^{1,2}$. No entanto, poucos meses após a pandemia entrar na América do Norte e na Europa, grandes séries de dados surgiram sobre os efeitos devastadores do vírus nesta população única de pacientes ${ }^{4,5,6}$.

O maior relatório da China incluiu dados de 13.077 pacientes com COVID-19, incluindo 232 que também tinham câncer. Esses pacientes apresentaram risco aumentado de COVID-19 grave (definido como frequência respiratória $\geq 30$ respirações por minuto, saturação de oxigênio de $93 \%$ ou menos em estado de repouso, razão de pressão parcial arterial de oxigênio para concentração de oxigênio $\leq 300 \mathrm{~mm} \mathrm{Hg}$, ou> $50 \%$ de progressão da lesão na imagem pulmonar dentro de 24-48 h) e morte, com taxas de $64 \%$ e $20 \%$ em pacientes com câncer em comparação com $32 \%$ e $11 \%$ em 518 pacientes estatisticamente pareados sem câncer, respectivamente ${ }^{7}$. Os dados deste estudo sugerindo que idade avançada, status de desempenho ECOG mais alto e doença em estágio mais avançado estão todos associados a um risco aumentado de mortalidade foram confirmados em outras séries, incluindo aquelas limitadas a pacientes com câncer de um único país, cidade ou sistema hospitalar na América do Norte e na Europa $4,5,6,8$. Pacientes do sexo masculino e de minorias étnicas também parecem ter 
maior probabilidade de serem diagnosticados com COVID-19 e de ter resultados piores ${ }^{9}$. Os dados diferiram em termos dos efeitos de intervenções específicas, incluindo quimioterapia, imunoterapia e cirurgia, nos resultados de pacientes com diagnóstico de COVID-19. É importante ressaltar que nem todos os pacientes com câncer parecem ter riscos iguais de mortalidade de COVID-19: pacientes com câncer de pulmão e aqueles com neoplasias hematológicas parecem ser particularmente suscetíveis. Lamentavelmente, nenhuma terapia única usada para tratar COVID-19 surgiu como benéfica para pacientes com câncer, e os dados do COVID-19 \& Cancer Consortium sugerem que a hidroxicloroquina em combinação com qualquer outro agente está associada a um risco aumentado de mortalidade, enquanto o remdesivir pode ser benéfico. No entanto, falta conhecimento sobre o momento ideal de administração do medicamento em relação ao início dos sintomas e a gravidade dos sintomas em pacientes no momento da administração do medicamento. Igualmente desesperador é que os pacientes com câncer parecem ter menos probabilidade de serem admitidos na unidade de terapia intensiva para escalonamento de cuidados ${ }^{4}$.

As implicações da pandemia para pacientes com câncer provavelmente serão sentidas nos próximos anos, com medo e apreensão de que as melhorias na mortalidade relacionada ao câncer alcançadas nos anos anteriores à pandemia serão compensadas pela interrupção dos programas de rastreamento e outros aspectos da cuidado do câncer. Em um modelo proposto por Norman Sharpless, do Instituto Nacional do Câncer dos Estados Unidos, prevê-se que um aumento de 1\% nas mortes por câncer colorretal e de mama ocorra na próxima década como resultado da interrupção do tratamento do câncer causada pela pandemia ${ }^{10}$. Este aumento previsto na mortalidade não leva em consideração atrasos na descoberta e no progresso como resultado de centros de câncer fechando temporariamente laboratórios de pesquisa e redirecionando recursos para o atendimento ao paciente, a suspensão temporária da inscrição para ensaios clínicos por empresas e instituições locais, e o fato de que estar disposto a viajar para um centro médico para receber tratamento é fundamental para a entrega e melhoria do atendimento ao paciente.

Notavelmente, os efeitos da pandemia no tratamento do câncer não foram totalmente negativos. A rápida evolução dos consórcios nacionais e globais para compreender melhor os efeitos do COVID-19 em pacientes com câncer e a transformação do tratamento do câncer em modelos mais centrados no paciente são estratégias que podem ser levadas adiante para melhorar 0 atendimento ao paciente. Além disso, o ritmo recorde no qual os ensaios clínicos avaliando as terapias potenciais para tratar, bem como prevenir, COVID-19 foram lançados define um novo padrão para a organização de futuros ensaios terapêuticos. Vários estudos envolvendo vacinas contra a SARS-CoV-2 estão atualmente em andamento e, com sorte, mitigarão 
os efeitos da pandemia em nossa comunidade global. Dado que os pacientes com câncer não estão incluídos em muitos desses ensaios, como eles irão responder a tais medidas preventivas permanece amplamente desconhecido. Mutações virais também podem ocorrer durante a transmissão e disseminação, levando a previsões de que o SARS-CoV2 permanecerá para sempre uma ameaça iminente para a comunidade oncológica. $\mathrm{O}$ que é importante lembrar é que o câncer em si é uma pandemia com> 18 milhões de pessoas diagnosticadas em todo o mundo. Muitas sociedades, incluindo ESMO e ASCO, estão fornecendo recomendações clínicas para o manejo de pacientes com câncer durante este período desafiador, reconhecendo que continuar a tratar nossos pacientes com sabedoria é fundamental para nosso papel como médicos e defensores de seus cuidados. O que é importante lembrar é que o câncer em si é uma pandemia com> 18 milhões de pessoas diagnosticadas em todo o mundo. Muitas sociedades, incluindo ESMO e ASCO, estão fornecendo recomendações clínicas para o manejo de pacientes com câncer durante este período desafiador, reconhecendo que continuar a tratar nossos pacientes com sabedoria é fundamental para nosso papel como médicos e defensores de seus cuidados. O que é importante lembrar é que o câncer em si é uma pandemia com> 18 milhões de pessoas diagnosticadas em todo o mundo. Muitas sociedades, incluindo ESMO e ASCO, estão fornecendo recomendações clínicas para o manejo de pacientes com câncer durante este período desafiador, reconhecendo que continuar a tratar nossos pacientes com sabedoria é fundamental para nosso papel como médicos e defensores de seus cuidados ${ }^{11,12}$.

\section{REFERÊNCIAS}

1. Chen, N. et al. Epidemiological and clinical characteristics of 99 cases of 2019 novel coronavirus pneumonia in Wuhan, China: a descriptive study. Lancet 395, 507-513 (2020).

2. Guan, W. J. et al. Clinical characteristics of coronavirus disease 2019 in China. $N$. Engl. J. Med. 382, 1708-1720 (2020).

3. The WHO Rapid Evidence Appraisal for COVID-19 Therapies (REACT) Working Group. Association between administration of systemic corticosteroids and mortality among critically ill patients with COVID-19: a meta-analysis. JAMA 324, 1330-1341 (2020). 
COVID-19 em pacientes com câncer: gerenciamento de uma pandemia dentro de uma pandemia. Figueira

4. Garassino, M. C. et al. COVID-19 in patients with thoracic malignancies (TERAVOLT): first results of an international, registry-based, cohort study. Lancet Oncol. 21, 914-922 (2020).

5. Kuderer, N. M. et al. Clinical impact of COVID-19 on patients with cancer (CCC19): a cohort study. Lancet 395, 1907-1918 (2020).

6. Robilotti, E. V. et al. Determinants of COVID-19 disease severity in patients with cancer. Nat. Med. 26, 1218-1223 (2020).

7. Tian, J. et al. Clinical characteristics and risk factors associated with COVID-19 disease severity in patients with cancer in Wuhan, China: a multicentre, retrospective, cohort study. Lancet Oncol. 21, 893-903 (2020).

8. Mehta, V. et al. Case fatality rate of cancer patients with COVID-19 in a New York hospital system. Cancer Discov. 10, 935-941 (2020).

9. Lee, L. Y. W. et al. COVID-19 prevalence and mortality in patients with cancer and the effect of primary tumour subtype and patient demographics: a prospective cohort study. Lancet Oncol. 21, 1309-1316 (2020).

10. Sharpless, N. E. COVID-19 and cancer. Science 368, 1290 (2020).

11. Paraguassu, Eber Coelho, et al. "Coronavirus and COVID-19: The latest news and views from the scientific community about the new coronavirus and COVID19." Brazilian Journal of Implantology and Health Sciences 2.3 (2020): 96-109.

12. Paraguassu, Eber Coelho. "COVID-19, a relação direta entre o capital, solidariedade e as vidas." Brazilian Journal of Implantology and Health Sciences 2.3 (2020): 01-04. 